\title{
Macrocyst Genetics in Polysphondylium pallidum, a Cellular Slime Mould
}

\author{
By D. FRANCIS \\ Department of Biological Sciences, University of Delaware, \\ Newark, DE I97I I, U.S.A.
}

(Received 28 January 1975)

SUMMARY

The discovery of two mating types in the cellular slime mould Polysphondylium pallidum is reported. Two developmental mutants produced in strains of opposite mating type but which do not proceed past the aggregation stage of development are capable of producing macrocysts. These macrocysts were viable and 5 to $10 \%$ germinated after 6 weeks of storage. When the macrocyst progeny were cloned, several classes of non-parental phenotypes were recovered.

\section{INTRODUCTION}

The cellular slime moulds are of increasing interest to developmental biologists, presenting as they do examples of eukaryotic cell differentiation and morphogenesis in a form which can be handled by the powerful methods of microbiology. Their potential value as experimental organisms has recently been increased by the findings that the long-known macrocyst stage represents a true zygote in at least one species (Erdos, Nickerson \& Raper, 1972; MacInnes \& Francis, 1974) and that the formation of macrocysts is controlled by a system of mating types in at least three species (Clark, Francis \& Eisenberg, 1973; Erdos, Raper \& Vogen, I973; Clark, 1974).

It has remained doubtful whether the macrocyst cycle will be useful in studying mutants of developmental interest. Many such mutants stop development before or during the stage of aggregation which ordinarily precedes macrocyst formation. A further difficulty is that macrocysts of several species are difficult to germinate. The present communication reports progress towards a resolution of these difficulties. Mating types are described for the first time in Polysphondylium pallidum. Macrocyst formation is demonstrated between two developmental mutants of this species. In addition, these macrocysts have been germinated and frequent non-parental phenotypes found among the progeny.

\section{METHODS}

Thirteen isolates of $P$. pallidum were obtained from the soil of the Morris Farm Forest of the University of Delaware, using a technique described by Clark et al. (1973).

Tests for mating reactions were performed in two ways. By the earlier procedure, the two strains to be tested were inoculated together with the bacterium Escherichia coli on to a Petri dish of cerophyll agar which was flooded with $10 \mathrm{ml}$ of Bonner's salt solution $(0.06 \%$ $\mathrm{NaCl}, 0.03 \% \mathrm{CaCl}_{2}, 0.075 \% \mathrm{KCl}$ ) and incubated in the dark for one week. With $P$. pallidum, only a few macrocysts form under these conditions, a fact which at first led us to suppose that this species is non-sexual (Clark et al. 1973). Later it was found that macrocysts formed much more densely if appropriate strains were mixed together with E. coli on a small area of 
Table I. Mating interactions among Morris Farm isolates of P. pallidum

All mixes were performed in triplicate. Isolates 1 to 3,7 , and 9 to 12 formed no macrocysts in any combination. No isolates formed macrocysts alone, even after several weeks.

$\begin{array}{lccccc} & \text { Pp5 } & \text { PpI3 } & \text { Pp4 } & \text { Pp6 } & \text { Pp8 } \\ \text { Pp5 } & 0 & 0 & + & + & + \\ \text { PpI3 } & & 0 & + & + & \pm \\ \text { Pp4 } & & & 0 & 0 & 0 \\ \text { Pp6 } & & & & 0 & 0 \\ \text { Pp8 } & & & & & 0\end{array}$

+ , Formation of many macrocysts; \pm , less dense or more erratic formation; 0 , no macrocysts formed.

an LP agar plate (0.I \% lactose, $0.1 \%$ peptone, I.5\% agar) and allowed to grow for one week in constant darkness without being flooded. This second procedure was used for all mating-type assays below.

Morphogenetic mutants were produced in two strains (Pp5 and Pp6) of $P$. pallidum with $N$-methyl- $N^{\prime}$-nitro- $N$-nitrosoguanidine (Aldrich Chemical Co.), using the method of Yanagisawa, Loomis \& Sussman (1967).

Growth rate was measured by inoculating the strain to be tested at one end of a streak of E. coli on cerophyll agar (Jones \& Francis, 1972). In all of the strains used, the feeding front of amoebae was visible as a sharp line behind which no bacteria were apparent and ahead of which no amoebae were apparent. The rate at which it advanced was independent of the heaviness of the bacterial streak, within wide limits. Two days after inoculation the position of the feeding front of amoebae was marked on the underside of the dish. The amoebae were then allowed to grow for an additional three to five days at 22 to $25{ }^{\circ} \mathrm{C}$ under dim light, after which the distance that the feeding front had advanced was measured.

\section{RESULTS}

\section{Mating types}

When the thirteen isolated strains were mixed in all possible pairs, macrocysts appeared only in certain cases (Table 1 ). The pattern of interaction permits the designation of two mating types: mating type I (represented by isolates PP5 and PpI3), and mating type II (isolates Pp4, Pp6 and Pp8). Isolates Pp5 and Pp6 have been deposited with the American Type Culture Collection as ATCC26659 and ATCC26658, respectively.

\section{Macrocyst germination}

A major difficulty is the germination of macrocysts. Nickerson \& Raper (1973) noted that light and low temperature stimulate germination in Dictyostelium mucoroides when applied three weeks or more after macrocyst formation. I found that macrocysts of $P$. pallidum which had formed on a wet plate of cerophyll agar did not germinate under any of several combinations of light, temperature, degree of dryness and length of storage that were tried. However, macrocysts which had formed in darkness on LP agar and were stored in darkness in sealed plastic bags at room temperature for 6 weeks did germinate at low frequency.

The procedure finally arrived at was to scrape several hundred macrocysts from the agar surface and suspend them in a few drops of sterile Bonner's salt solution in a depression slide under room light and temperature. Three days later the macrocysts were removed to 
D. FRANCIS
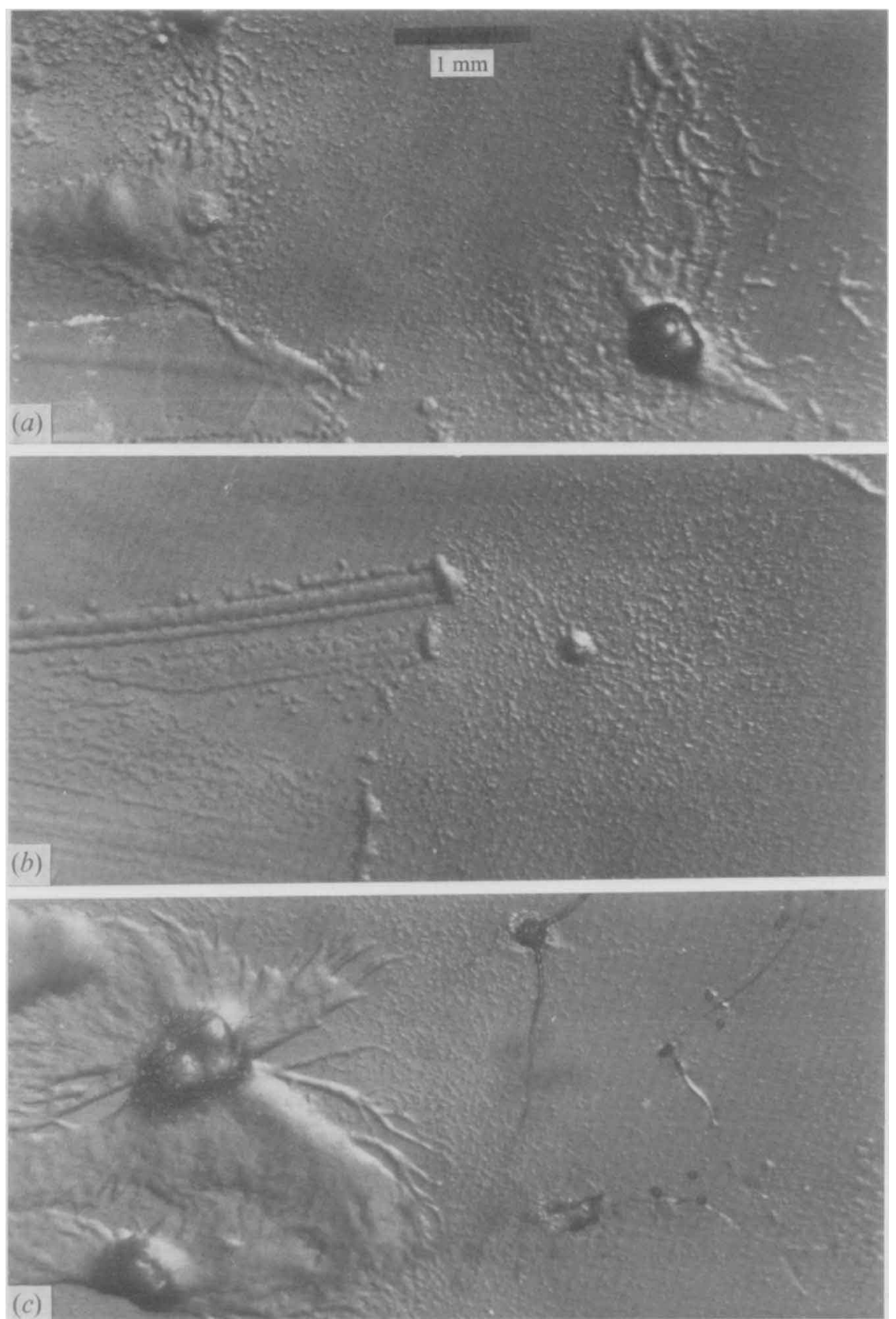

Fig. 1. Phenotypic appearance of three strains of $P$. pallidum, growing on cerophyll agar. (a) Mutant B; (b) mutant A; (c) Pp5. The area to the left of each photograph consists of uneaten bacteria, upon which the front of feeding amoebae advances from the right. Terminal stages of development appear in the right-hand part of the pictures. 
Table 2. Linear growth rate of parent strains and of progeny clones recovered from macrocyst hatchings

$\begin{array}{lc}\quad \text { Line } & \text { Growth rate (mm/day) } \\ \text { Parents } & \\ \text { Mutant A } & 1 \cdot 0-1 \cdot 1 \text { (I0 clones) } \\ \text { Mutant B } & 2 \cdot 6-3 \cdot 9 \text { (I0 clones) } \\ \text { Pp6 } & 2 \cdot 4-3 \cdot 9 \text { (I0 clones) } \\ \text { Pp5 } & 4 \cdot 1-4 \cdot 8 \text { (I0 clones) } \\ \text { Progeny } & \\ \text { Type A, mating type II } & 0 \cdot 7-2 \cdot 5 \text { (I0 clones) } \\ \text { Type A, mating type I } & 0 \cdot 7-2 \cdot 2 \text { (9 clones) } \\ \text { Type B, mating type I } & 2 \cdot 2-5 \cdot 2 \text { (23 clones) } \\ \text { Wild type, mating type I } & 2 \cdot 0-4 \cdot 0 \text { (I3 clones) }\end{array}$

a sterile cup made of stainless steel wire mesh of $25 \mu \mathrm{m}$ aperture, and thoroughly rinsed with sterile salt solution to remove amoebae, spores and microcysts which may have been clinging to the macrocysts; with macrocysts of $D$. mucoroides such rinsing is completely effective (MacInnes \& Francis, 1974). The macrocysts were then returned to the sterile depression slide. Three or four days later, 5 to $10 \%$ of the macrocysts had become thinwalled and empty, and many amoebae were observed in the depressions.

\section{Mutants}

Two morphogenetic mutants were used in crosses. Mutant B is a mutant of $\mathrm{Pp}_{5}$, and when grown at room temperature on $E$. coli spread on cerophyll agar develops almost normally until the stage of late aggregation when large tight mounds of cells are present (Fig. I $a$ ). Under ideal conditions, as when the plate is inverted over a layer of charcoal, many of the mounds continue development to fruiting bodies with very thin, short, unbranched stalks bearing spore heads with viable spores of normal appearance. The remaining masses persist unchanged for several days. Under less ideal conditions, only aggregated masses appear. Growth rate on E. coli is near that of wild type (Table 2). Mutant A is a mutant of Pp6 that aggregates with the appearance of fewer streams than usual and forms small loose mounds (Fig. I b). The mounds break up into scattered amoebae a few hours after they form, and never develop to fruiting bodies. Growth rate is much less than that of wild type (Table 2).

\section{Backcrosses}

Cells of each morphogenetic mutant were mixed with cells of a wild-type strain of opposite mating type (Pp5 or Pp6). Macrocysts were germinated and the amoebae resulting from a group of several hundred macrocysts were cloned on to cerophyll plates uniformly spread with $E$. coli. At io to 20 amoebae per plate, the cloning efficiency averaged $80 \%$. The technique of cloning progeny from single macrocysts employed by MacInnes \& Francis (1974) with $D$. mucoroides could not be used with $P$. pallidum because of the low frequency of germination. All progeny of each cross were identifiable as having either mutant or wild-type morphology (Table 3). Both mating types were present in progeny of both phenotypes, demonstrating that genetic exchange had occurred. The progeny of $\mathbf{B}$ phenotype (the phenotype of mutant B) had aggregated masses of variable size. These variations did not fall into two or more discrete classes, but ranged over a continuous series of types. 


\section{Table 3. Phenotypes of progeny clones of backcrosses}

\begin{tabular}{|c|c|c|c|c|c|c|c|}
\hline \multirow[b]{3}{*}{ Mutant $\mathrm{A} \times \mathrm{Pp}_{5}$} & \multirow{2}{*}{$\begin{array}{c}\text { No. } \\
\text { of clones }\end{array}$} & \multirow[b]{2}{*}{ Percentage } & \multicolumn{4}{|c|}{ Mating type } & \multirow{2}{*}{$\begin{array}{l}\text { Total } \\
\text { tested }\end{array}$} \\
\hline & & & I & II & $\mathrm{O}$ & $\mathbf{S}$ & \\
\hline & & & & & & & \\
\hline Wild type & 259 & 78 & $54^{\mathrm{P}}$ & 5 & 3 & 0 & 62 \\
\hline A & 75 & 22 & 8 & $4 \mathrm{I}^{\mathrm{P}}$ & 0 & 0 & 49 \\
\hline B & 0 & o & & & & & \\
\hline \multicolumn{8}{|l|}{ Mutant $\mathrm{B} \times \mathrm{Pp} 6$} \\
\hline Wild type & 148 & 62 & 7 & $20^{\mathrm{P}}$ & 0 & 0 & 27 \\
\hline A & 0 & o & & & & & \\
\hline B & $9 \mathrm{I}$ & 38 & $26^{\mathrm{P}}$ & 8 & 0 & 0 & 34 \\
\hline
\end{tabular}

Table 4. Phenotypes of progeny clones of dihybrid cross

For symbols, see Table 3.

\begin{tabular}{|c|c|c|c|c|c|c|c|}
\hline \multirow[b]{2}{*}{ Mutant $\mathrm{A} \times$ mutant $\mathrm{B}$} & \multirow[b]{2}{*}{$\begin{array}{l}\text { No. } \\
\text { of clones }\end{array}$} & \multirow[b]{2}{*}{ Percentage } & \multicolumn{4}{|c|}{ Mating type } & \multirow[b]{2}{*}{$\begin{array}{l}\text { Total } \\
\text { tested }\end{array}$} \\
\hline & & & I & II & $\mathrm{O}$ & $\mathrm{S}$ & \\
\hline $\begin{array}{l}\text { Wild type } \\
\text { A }\end{array}$ & $\begin{array}{r}34 \\
122\end{array}$ & $\begin{array}{r}8 \\
29\end{array}$ & $\begin{array}{l}21 \\
15\end{array}$ & $\begin{array}{c}7 \\
22^{P}\end{array}$ & $\begin{array}{l}\circ \\
3\end{array}$ & $\begin{array}{l}\circ \\
\circ\end{array}$ & $\begin{array}{l}28 \\
40\end{array}$ \\
\hline B & 265 & 63 & $49^{P}$ & I & 0 & I & $5 \mathrm{I}$ \\
\hline
\end{tabular}

\section{Dihybrid cross}

When cells of mutant B were mixed with cells of mutant A on LP agar, many macrocysts appeared within one week. A very few microcysts formed and a few stunted fruiting bodies of B phenotype developed as well. These mutants did not interact synergistically to form wild-type fruits. Clones from germinated macrocysts were found to be of three morphological types - wild type, $A$ and $B$ - in the proportions given in Table 4.

Since some of the type A clones (with phenotype of mutant A) appeared at first to be fast growing, unlike the parent mutant A, the growth rate of some of these was compared with that of wild type under standard conditions and it was found that all were in fact slow growing. Similarly, type B clones resembled the mutant B parent in growth rate (Table 2).

A number of clones of each type were tested for mating type by mixing with Pp5 and Pp6 on LP agar. Both mating types appeared in several morphological classes of progeny, but in variable proportions (Table 4).

As encountered in the backcrosses, there was considerable morphological variation within the class of type B progeny. Some of these (BI) differed from the original mutant phenotype in having more numerous and smaller aggregates. The character is stable through mitosis, for when several BI progeny were recloned, all of the resulting clones were of the BI phenotype. Two BI clones were backcrossed against the parental wild type of appropriate mating type. The progeny were of wild type, B and BI phenotype and included intermediates between B and BI. This suggests that the expression of the primary mutation is modified by a number of genes.

Since mutant A stops development at an earlier stage than mutant $\mathrm{B}$, it was suspected that the class of A progeny of the dihybrid cross might contain double mutants. To search for these, seven progeny clones of A phenotype were crossed against the wild-type parent of 
Table 5. Phenotypes of progeny clones of $F_{2}$ crosses

For symbols, see Table 3.

$\mathrm{F}_{2}$ cross

(I) $\mathrm{A} \times \mathrm{Pp} 6$

Wild type

A

(2) $\mathrm{A} \times$ Pp6

Wild type

A

(3) $\mathrm{A} \times$ Pp5

Wild type

B

(4) A $\times$ Pp5

Wild type

A

(5) $\mathrm{A} \times$ Pp5

Wild type

A

(6) A $\times$ Pp 6

Wild type
B

(7) $\mathrm{A} \times \mathrm{Pp} 5$

$\begin{array}{lrr}\text { Wild type } & 224 & 40 \\ \text { A } & 282 & 51 \\ \text { B } & 53 & 9\end{array}$

$\begin{array}{rr}171 & 71 \\ 69 & 29 \\ 0 & 0\end{array}$

$\begin{array}{rr}78 & 74 \\ 27 & 26 \\ 0 & 0\end{array}$

$\begin{array}{rr}77 & 55 \\ 62 & 45 \\ 0 & 0\end{array}$

$\begin{array}{cr}\begin{array}{c}\text { No. } \\ \text { of clones }\end{array} & \text { Percent } \\ & \\ \text { I45 } & 55 \\ \text { I } 6 & 45 \\ \text { O } & 0\end{array}$

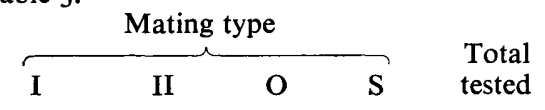

55

45
0

I I $^{\mathrm{P}}$

${ }_{10}^{\mathrm{I}}$

I
0

0

13

0

$\begin{array}{rr}109 & 95 \\ 6 & 5 \\ 0 & 0\end{array}$

$\begin{array}{rr}147 & 28 \\ 318 & 59 \\ 72 & 13 \\ & \\ 224 & 40 \\ 282 & 51 \\ 53 & 9\end{array}$

$8^{P}$
4
10

1
4

$\begin{array}{rr}0 & 10 \\ 0 & 7 \\ 0 & 32\end{array}$

I I

2

0

I6

appropriate mating type. In two crosses, clones of B phenotype appeared in the progeny (Table 5). This result indicates that the mutated gene of mutant $A$ is epistatic to that of mutant B.

\section{DISCUSSION}

Genetic exchange. The recovery of wild-type progeny from the dihybrid cross between two morphogenetic mutants shows that exchange of genetic material occurs in the macrocyst and agrees with the idea that the macrocyst represents a zygote, as suggested for D. mucoroides (MacInnes \& Francis, 1974). However, several alternative possibilities must be considered.

Firstly, these wild types might represent back mutations. That this cannot be so is shown by absence of revertants when old cultures of the parent strains were cloned. Mutant B showed no wild types in 277 clones, and mutant A showed none in 468 clones. A second possibility is that the wild-type clones represent either heterokaryons or heterozygous diploids stemming from a fusion of two different parental cells. Such a phenomenon has been reported by other workers on the cellular slime moulds (Katz \& Sussman, 1972; Yanagisawa \& Yamada, I972), but it does not appear likely here. Heterogenomic strains might be expected to exhibit both mating types and therefore to be selfers. In fact, however, all the wild-type clones except three were of one or the other mating type (Table 4). In 
Table 6. Spore sizes of wild-type strains and of wild-type progeny of a cross of mutant $B$ by mutant $A$

\begin{tabular}{lcccc}
\cline { 2 - 3 } & \multicolumn{2}{c}{ Wild-type strains } & Pp28s & $\begin{array}{c}\text { Wild-type progeny } \\
\text { (26 clones) }\end{array}$ \\
Mean length of & 6.32 & 6.62 & 9.64 & $6.26-7.23$ \\
spore $(\mu \mathrm{m})$ & 0.49 & 0.72 & 0.43 & $0.3-0.59$
\end{tabular}

addition, these wild-type progeny do not exhibit the large spore size which is characteristic of the diploid lines of at least one other species of cellular slime mould (Sussman \& Sussman, 1962; Sackin \& Ashworth, 1969). Instead, their spore sizes are very near to those of the parental wild-type strains (Table 6). The existence of wild-type strains of P. pallidum, which have the properties postulated of a diploid heterozygous for mating type, is proved by the isolation from nature of strain Pp28s which has large spores (Table 6) and which 'selfs', or forms macrocysts in a pure clone ( $R$. Eisenberg, unpublished observation).

Finally, diploid lines of slime moulds are often unstable and degenerate to aneuploid and haploid strains (Brody \& Williams, 1974). Some of these haploid end-products exhibit the parental phenotypes of the cross. When a total of 1547 such clones made from twelve different wild-type progeny were inspected, however, no A or B clones were found.

Together, the evidence suggests that the wild-type progeny are not diploids or heterokaryons. It remains possible, however, that they represent haploid strains which have been generated by a parasexual cycle (i.e., from a heterodiploid line which decays through aneuploidy to haploidy) rather than by a true sexual cycle, which includes meiosis of a zygote. If so, the decay of the diploid must be completed very early, during the three days when the macrocysts are germinating, because the data given above indicate that clones from amoebae which appear at that time are already homogeneous for both mating type and morphological phenotype.

Phenotypic ratios. Examination of the progeny of the backcrosses between each mutant and wild type suggests that each mutant carries a lesion in only a single locus. The demonstration that some progeny of the dihybrid cross contain both mutations indicates that the mutated gene of mutant $\mathbf{A}$ is epistatic to that in mutant $B$. Therefore the progeny of the dihybrid cross are expected to occur in the ratio I wild type:2 A:I B, supposing that the two mutant loci are unlinked. If the loci are closely linked, A and B phenotypes should appear in nearly equal numbers and wild type should be rare in the progeny. Neither of these expectations is precisely fulfilled by the data shown in Table 4. Wild type are indeed rare, but $\mathrm{B}$ phenotypes are much more frequent than $\mathrm{A}$.

In the backcross of doubly mutant progeny to wild-type parent, the expected ratios are I wild type:2 A: I B in the absence of linkage; or, alternatively, few B, few A and wild type present in large, nearly equal numbers if linkage is present. Again the second case is more nearly fulfilled, but not exactly so.

Several factors might produce abnormal phenotypic ratios in the progeny. Firstly, contamination of macrocysts with parental cells, which did not enter macrocysts but which weathered washing steps and the storage period, is conceivable. When individual macrocysts of $D$. mucoroides were germinated following a similar procedure, inspection of the progeny showed that no contamination occurred (MacInnes \& Francis, 1974); this suggests that it may also be infrequent in the case of $P$. pallidum. Nevertheless, parental categories of progeny in Tables 3 and 4 may be artificially large from this cause. Another factor affecting 
ratios is the differential division rate of the several phenotypic classes. The slow growth rate of A clones (Table 2) quite probably reflects a low division rate. This factor would depress the numbers of all A classes. A third possibility is that some zygotes may result from fusion of two gametes of the same mating type. The result would be to overload the parental classes of progeny. It will be possible to distinguish among these causes if the frequency of germination can be increased to the level where the progeny of single macrocysts can be examined.

Control of mating type. All of the progeny scored for mating type fall into types I or II, with the exception of rare clones which were either asexuals or 'selfers' (Tables 3 and 4). It is not known whether these exceptions are real, or whether they represent impure clones which act as 'selfers' or clones whose mating was prevented by bacterial contamination. (The latter occurrence has been observed to prevent macrocyst formation between wildtype strains Pp5 and Pp6.)

Mating type I appears to occur more frequently in the progeny than mating type II (Tables 4 and 5). This imbalance cannot wholly be caused by contamination of the progeny with parentals of the mating type I class, since it occurs also in the class of wild-type progeny of the dihybrid cross which consists entirely of non-parentals (Table 4). This finding suggests that mating type is not simply controlled by two alleles at a single locus. The situation may possibly be similar to cases in the ciliated Protozoa, where mating type is controlled by multiple loci, or by post-genomic factors such as parental cytoplasm and environmental influences following zygote formation (Grell, 1973). These possibilities remain to be investigated.

I thank David Sheppard and Hubert Ling who improved the manuscript. The work was supported by USPHS grant No. GM 19003.

\section{REFERENCES}

Brody, T. \& Williams, K. L. (1974). Cytological analysis of the parasexual cycle in Dictyostelium discoideum. Journal of General Microbiology 82, $37 \mathrm{I}-383$.

Clark, M. A. (1974). Syngenic divisions of the cellular slime mold, Polysphondylium violaceum. Journal of Protozoology 21, 755-757.

Clark, M. A., Francis, D. \& Eisenberg, R. (1973). Mating types in cellular slime molds. Biochemical and Biophysical Research Communications 52, 672-678.

Erdos, G. W., Nickerson, A. W. \& RAPER, K. B. (1972). Fine structure of macrocysts in Polysphondylium violaceum. Cytobiologie 6, $35 \mathrm{I}-366$.

ERdos, G. W., RAPER, K. B. \& Vogen, L. K. (1973). Mating types and macrocyst formation in Dictyostelium discoideum. Proceedings of the National Academy of Sciences of the United States of America 70, 1828I 830 .

Grell, K. G. (1973). Protozoology, 2nd ed. New York: Springer-Verlag.

Jones, W. R., III \& Francis, D. (I972). The action spectrum of light induced aggregation in Polysphondylium pallidum, and a proposed general mechanism for light response in the cellular slime molds. Biological Bulletin 142, 46I-469.

Katz, E. R. \& Sussman, M. (1972). Parasexual recombination in Dictyostelium discoideum: selection of stable diploid heterozygotes and stable haploid segregants. Proceedings of the National Academy of Sciences of the United States of America 69, 495-498.

MacInNes, M. \& Francis, D. (1974). Meiosis in Dictyostelium mucoroides. Nature, London 251, 32 I-323.

Nickerson, A. W. \& RAPER, K. B. (I973). Macrocysts in the life cycle of the Dictyosteliaceae. II. Germination of the macrocysts. American Journal of Botany 6o, 247-254.

SACKIN, M. J. \& Ashworth, J. (1969). An analysis of the distribution of volumes amongst spores of the cellular slime mould Dictyostelium discoideum. Journal of General Microbiology 59, 275-284. 
Sussman, M. \& SUSSMAN, R. R. (1962). Ploidal inheritance in Dictyostelium discoideum: stable haploid, stable diploid and metastable strains. Journal of General Microbiology 28, 4I7-429.

Yanagisawa, K., Loomis, W. F., Jưn. \& Sưssman, M. (1967). Developmental regulation of the enzyme UDP-galactose polysaccharide transferase. Experimental Cell Research 46, 328-334.

YANAGisawa, K. \& YAMADA, T. (1972). Appearance of wild type from mixed culture of two aggregateless mutants in the cellular slime mold Dictyostelium discoideum. Cytologia 36, 534-538. 